\title{
Construção e validação de instrumento para coleta de dados de enfermagem em ambulatório de quimioterapia
}

\author{
Construction and validation of instrument for nursing data collection in chemotherapy \\ ambulatory
}
Construcción y validación de instrumento para recogida datos de enfermería en quimioterapia ambulatoria

Igor Guerra Cheloni ${ }^{1 *}$, Cristiane Chaves de Souza ${ }^{1}$, José Victor Soares da Silva ${ }^{1}$, Patrícia de Oliveira Salgado ${ }^{1}$, Tânia Couto Machado Chianca ${ }^{2}$.

\section{RESUMO}

Objetivo: Construir e validar um instrumento para coleta de dados de enfermagem em um ambulatório de quimioterapia, fundamentado na teoria das Necessidades Humanas Básicas de Wanda Horta (NHB). Métodos: Pesquisa metodológica. A coleta de dados se deu por meio eletrônico, em quatro etapas: revisão integrativa da literatura para identificar os indicadores empíricos do que deve ser avaliado na população estudada, validação dos indicadores junto a especialistas, construção do instrumento de coleta de dados e avaliação de especialistas, e validação da versão final do instrumento junto a especialistas. Resultados: $\mathrm{Na}$ revisão integrativa foram identificados 107 indicadores empíricos. Destes, 83 foram validados. 58 novos indicadores foram sugeridos na segunda etapa. Estes foram agrupados de acordo com os constructos de avaliação de cada NBH para a construção da versão preliminar do instrumento. Nesta etapa, os especialistas sugeriram 37 novas modificações na versão preliminar do instrumento. Na última etapa, foi elaborada a versão final do instrumento e nova submissão para avaliação dos especialistas. Após 24 novas modificações, chegou-se à versão final do instrumento de coleta de dados. Conclusão: $O$ instrumento construído contribuirá para a ressignificação do trabalho do enfermeiro no local em estudo, e para uma assistência sistematizada a esta clientela.

Palavras-chave: Enfermagem oncológica, Processo de enfermagem, Quimioterapia.

\begin{abstract}
Objective: Build and validate an instrument for collecting nursing data in a chemotherapy ambulatory, based on the Wanda Horta theory of Basic Human Needs (BHN). Methods: Methodological research. Data collection took place electronically, in four stages: integrative literature review to identify the empirical indicators of what should be evaluated in the population studied, validation of the indicators with specialists, construction of the data collection instrument and evaluation of specialists, and validation of the final version of the instrument with specialists. Results: In the integrative review, 107 empirical indicators were identified. From these ones, 83 were validated. In the second stage 58 new indicators were suggested. These ones were grouped according to the assessment constructs of each BHN for the construction of the preliminary version of the instrument. At this stage, the experts suggested 37 new modifications to the preliminary version of the instrument. In the last stage, the final version of the instrument was elaborated and there was a new submission for evaluation by the specialists. After 24 new modifications, the final version of the data collection instrument was reached. Conclusion: The built instrument will contribute to the re-signification of the nurse's work and to a systematic assistance to this clientele in the place under study.
\end{abstract}

Keywords: Oncologic nursing, Nursing process, Chemotherapy.

1 Universidade Federal de Viçosa (UFV), Viçosa - MG. *E-mail: igorcheloni@yahoo.com.br

2 Universidade Federal de Minas Gerais (UFMG), Belo Horizonte - MG. 


\section{RESUMEN}

Objetivo: Construir y validar un instrumento para la recolección de datos de enfermería en una consulta externa de quimioterapia, basado en la teoría de Necesidades Humanas Básicas de Wanda Horta (NHB). Métodos: Investigación metodológica. La recolección de datos se realizó de manera electrónica, en cuatro etapas: revisión integradora de la literatura para identificar los indicadores empíricos de lo que se debe evaluar en la población estudiada, validación de los indicadores con especialistas, construcción del instrumento de recolección de datos y evaluación de especialistas, y validación de la versión final del instrumento con especialistas. Resultados: En la revisión integradora se identificaron 107 indicadores empíricos. De estos, 83 fueron validados. Se sugirieron 58 nuevos indicadores en la segunda etapa. Estos se agruparon de acuerdo a los constructos de evaluación de cada NBH para la construcción de la versión preliminar del instrumento. En esta etapa, los expertos sugirieron 37 nuevas modificaciones a la versión preliminar del instrumento. En la última etapa se elaboró la versión final del instrumento y se presentó un nuevo envío para evaluación por parte de los especialistas. Luego de 24 nuevas modificaciones, se alcanzó la versión final del instrumento de recolección de datos. Conclusión: El instrumento construido contribuirá a la resignificación del trabajo de la enfermera en el lugar de estudio y a una asistencia sistemática a esta clientela.

Palabras clave: Enfermería oncológica, Proceso de enfermería, Quimioterapia.

\section{INTRODUÇÃO}

Para um cuidado efetivo, é fundamental que a assistência de enfermagem ofereça qualidade e segurança, e seja sustentada por ações baseadas em uma metodologia de cuidado, como o Processo de Enfermagem $\mathrm{PE}$. Este é um conjunto de ações sistematizadas e inter-relacionadas, executadas segundo um determinado modo de pensar para assistência integral ao paciente, seguindo etapas metodológicas, responsáveis por um contínuo processo de raciocínio e julgamento clínico que orienta as ações de enfermagem (VIDIGAL PD, et al., 2017).

O Processo de Enfermagem se operacionaliza em etapas que variam de acordo com autores quanto ao número e à terminologia (TANNURE MC e GONÇALVES AM, 2019). Segundo a Resolução COFEN № 358/2009, deve ser realizado em cinco etapas, a primeira etapa do processo de Enfermagem consiste em um processo deliberado, sistemático e contínuo, realizado com o auxílio de métodos e técnicas variadas para obtenção de informações sobre a pessoa, família ou coletividade humana e sobre suas respostas em um dado momento do processo saúde e doença (COFEN, 2009). Para guiar a consulta do Enfermeiro, têm sido elaborados instrumentos de coleta de dados, pautados em um referencial teórico de cuidados, para a seleção de informações que irão substanciar as etapas subsequentes do Processo de Enfermagem (RODRIGUES SMN, 2017).

Dentre as clientelas assistidas pela equipe de Enfermagem, destaca-se o paciente oncológico. O câncer é um problema de saúde pública, com mais de 18 milhões de casos em 2018 e previsão de 29 milhões para 2040 (BRAY F, et al., 2018). A estimativa para o triênio 2020-2022 é de 625 mil casos novos de câncer no Brasil (INCA, 2019). Uma das possibilidades de manejo terapêutico em pacientes oncológicos, é a quimioterapia, que consiste no uso de substâncias químicas isoladas ou combinadas para tratar neoplasias malignas, agindo diretamente no crescimento e divisão das células.

É uma modalidade de tratamento sistêmico, que contrasta com a cirurgia e radioterapia de atuação localizada (BONASSA EMA e GATO MIR, 2012). Em virtude dos efeitos colaterais físicos e emocionais causados pelo tratamento, sistematizar a assistência de enfermagem utilizando como metodologia de cuidado o processo de enfermagem a estes pacientes torna-se essencial para a qualidade do cuidado. Para tanto, o primeiro passo é a realização da coleta de dados de enfermagem. Durante a prática clínica enquanto enfermeiro oncológico de um ambulatório de quimioterapia, percebeu-se que a assistência do Enfermeiro se restringia a seguir os protocolos de infusão dos quimioterápicos, carecendo do olhar holístico pautado em um referencial teórico de cuidado. Esse modo de organizar o processo de trabalho limitava o cuidado do enfermeiro, gerando inquietações sobre sua identidade profissional naquele espaço. 
A partir de então, delineou-se este trabalho com vistas à implantação da primeira etapa do processo de Enfermagem no ambiente em estudo. Assim, delineou-se este estudo com objetivo de construir e validar um instrumento para coleta de dados de enfermagem em um ambulatório de quimioterapia, fundamentado na teoria das Necessidades Humanas Básicas de Wanda Horta.

\section{MÉTODOS}

Trata-se de estudo do tipo metodológico, realizado entre março de 2019 e junho de 2020 em uma universidade pública federal, em parceria com um hospital de referência para tratamento oncológico.

O estudo foi realizado em 4 etapas, seguindo modelo metodológico de pesquisa semelhante realizado por Ramalho JMN (2010) para construção e validação de instrumento de coleta de dados de Enfermagem em pacientes adultos de uma Unidade de Terapia Intensiva (Figura 1).

Figura 1 - Fluxograma com a descrição das etapas metodológicas da pesquisa.

Etapa 1: Identificação na literatura dos indicadores empíricos de
avaliação das Necessidades Humanas Básicas em pacientes oncológicos

Etapa 2: Validação dos indicadores empíricos junto a um comitê de especialistas

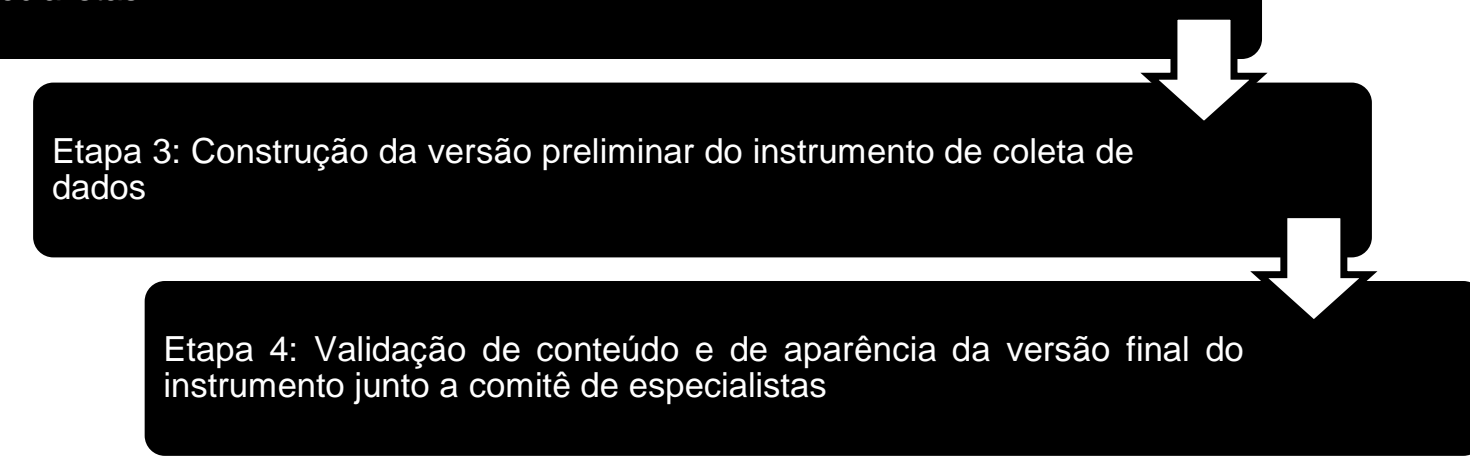

Fonte: Cheloni IG, et al., 2020.

A primeira etapa do estudo foi realizada no período de março a abril de 2019 , e consistiu em revisão integrativa da literatura, guiada pela questão norteadora: "Quais são as publicações na literatura nacional e internacional acerca dos indicadores empíricos que indicam alterações nas necessidades humanas em pacientes oncológicos submetidos a quimioterapia ambulatorial?"Por indicadores empíricos entendem-se as manifestações, observadas ou mensuradas, que evidenciam no cliente necessidades humanas básicas afetadas (SOUZA APMA, et al., 2009).

A busca na literatura foi realizada utilizando os portais Periódicos Capes e Google Acadêmico, e as bases de dados Cinahl, LILACS, Biblioteca Cochrane, e PubMed. A pesquisa ocorreu mediante a utilização dos descritores controlados contidos nos Descritores em Ciência da Saúde (DeCS) da Biblioteca Virtual em Saúde (BVS): enfermagem, processos de enfermagem, quimioterapia, enfermagem oncológica, diagnósticos de enfermagem, necessidades biológicas, necessidades psicossociais, necessidades espirituais. Foi usado 0 operador booleano "and" para a combinação dos descritores.

Os critérios utilizados para a seleção da amostra foram: artigos, dissertações ou teses publicadas na literatura nacional e internacional cujo foco tenha sido o estudo da construção e validação de instrumentos de coleta de dados para enfermagem, não sendo filtrado o ano e a linguagem de publicação. A extração dos dados seguiu formulário construído conforme metodologia descrita por Mendes KDS, et al., (2008), contendo: tipo de publicação, autor(es), país/ano de publicação, idioma, objetivo principal do estudo, tipo de estudo e número de sujeitos participantes, conclusão e indicadores empíricos extraídos. Após extração dos indicadores empíricos identificados nos trabalhos elencados, foram eliminados os indicadores duplicados. 
Os dados foram analisados por pares e validados por um terceiro pesquisador segundo os conteúdos apresentados pelos artigos, utilizando estatística descritiva. Cada estudo foi avaliado quanto ao nível de evidência de I a VII, de acordo com o desenho metodológico (GALVÃO CM, 2014). Para apresentação dos resultados, os indicadores empíricos foram agrupados de acordo com os constructos de avaliação das NHB, com auxílio do programa Microsoft Excel® 2013, seguindo o referencial teórico de Wanda Horta, e revisão das definições conceituais proposta por Benedet SA e Bub MBC (2001), utilizando a técnica de mapeamento cruzado. Para cada indicador, procedia-se à leitura da definição operacional de cada NHB e, na sequência, era feita a alocação dos indicadores empíricos conforme a NHB a que pertencia.

A segunda etapa do estudo foi realizada no período de maio a junho 2019. Os indicadores empíricos identificados na Etapa 1 do estudo passaram foram validados quanto ao conteúdo junto a um grupo de especialistas. Foram incluídos no estudo aqueles com pontuação mínima de cinco, utilizando o sistema de pontuação de especialistas proposto por Fehring RJ (1987), adaptado para esta pesquisa (Quadro 1).

Quadro 1 - Critérios de Fehring que foram utilizados para seleção dos especialistas que compuseram a amostra do estudo.

\begin{tabular}{|l|c|}
\hline \multicolumn{1}{|c|}{ Critério } & Pontuação \\
\hline Prática clínica de pelo menos 1 ano de duração na área de enfermagem oncológica. & 4 \\
\hline $\begin{array}{l}\text { Doutorado - temática central em construção e validação de instrumentos de coletas de } \\
\text { dados baseados em teorias de enfermagem OU temática de estudo envolvendo } \\
\text { assistência de Enfermagem a pacientes oncológicos. }\end{array}$ & 2 \\
\hline Mestrado - dissertação com conteúdo relevante dentro da área Enfermagem Oncológica. & 1 \\
\hline $\begin{array}{l}\text { Especialização em Enfermagem Oncológica com Prática clínica de pelo menos 1 ano de } \\
\text { duração na área de enfermagem oncológica }\end{array}$ & 3 \\
\hline $\begin{array}{l}\text { Especialização em Enfermagem Oncológica sem prática clínica na área, ou com prática } \\
\text { clínica inferior a 1 ano de duração na área de enfermagem oncológica: 1 ponto. }\end{array}$ & 1 \\
\hline Pesquisa (com publicações) na área de Enfermagem Oncológica. & 1 \\
\hline $\begin{array}{l}\text { Publicações em revistas Qualis Capes A1, A2 ou B1 na Enfermagem envolvendo a } \\
\text { temática "Processo de Enfermagem" ou “Enfermagem oncológica" ou "Assistência de } \\
\text { Enfermagem em Ambulatórios de Quimioterapia" }\end{array}$ & 2 \\
\hline Ser Enfermeiro, com diagnóstico de câncer, submetido a quimioterapia ambulatorial. & 2 \\
\hline
\end{tabular}

Fonte: Fehring RJ, 1987. Dados coletos por Cheloni IG, et al., 2020.

Os especialistas foram recrutados via contato com integrantes da Associação Brasileira de Enfermagem Oncológica, da Associação Brasileira de Enfermagem em Oncologia e Oncohematologia e enfermeiros atuantes na instituição alvo da pesquisa. O convite foi enviado por e-mail para 261 especialistas. Destes, 28 aceitaram em participar do estudo, e dentre estes, 14 atenderam aos critérios de inclusão, superando assim o número mínimo de avaliadores recomendado por Lynn MR (1986). Para os incluídos no estudo, foi enviado por meio eletrônico (e-mail) carta convite esclarecendo o objetivo do estudo, o Termo de Consentimento Livre e Esclarecido (TCLE), e o instrumento de coleta dos dados.

No instrumento de coleta dos dados, os indicadores empíricos foram agrupados de acordo com os constructos de avaliação das NHB, seguindo o referencial teórico de Wanda Horta, e revisão das definições conceituais proposta por Benedet SA e Bub MBC (2001). Para cada indicador empírico, os especialistas julgavam o item como relevante ou não relevante de ser avaliado em pacientes submetidos à quimioterapia ambulatorial. Para cada indicador empírico, havia também espaço para sugestões de alteração do nome do indicador de acordo com o mais comumente utilizado na prática clínica, e outro para sugestão de inclusão de outros indicadores que os especialistas considerassem essencial para avaliação daquela necessidade humana básica.

Nesta etapa, foram considerados válidos os indicadores apontados como relevantes por $80 \%$ ou mais dos especialistas, conforme critérios estabelecidos por Lynn MR (1986). Também foram considerados para inclusão na versão preliminar do instrumento de coleta de dados todos os indicadores sugeridos pelos especialistas e não identificados na literatura. Estes foram apreciados novamente pelos especialistas na terceira etapa do estudo. 
A terceira etapa do estudo foi realizada entre agosto e outubro de 2019. Para a construção e formatação da primeira versão do instrumento de coleta de dados, foram considerados os indicadores empíricos com o IC $\geq 80 \%$ e todos os indicadores sugeridos pelos especialistas na segunda etapa da coleta dos dados.

Os indicadores empíricos validados na segunda etapa do estudo e os sugeridos para inclusão foram agrupados em cada NHB, seguindo a definição operacional de cada necessidade proposta por Benedet SA e Bub MBC (2001), e enviados para nova apreciação pelo mesmo grupo de especialistas, em uma forma preliminar do instrumento de coleta de dados.

A exemplo do que foi feito na etapa 2, havia novamente um espaço para sugestão de inclusão ou exclusão de algum item que considerasse mais adequado para utilizar na prática clínica. Nesta etapa, 13 especialistas responderam à avaliação.

A quarta etapa do estudo foi realizada em junho de 2020. Nesta etapa, o instrumento de coleta de dados construído a partir das modificações sugeridas pelos especialistas da versão preliminar do instrumento na etapa 3 do estudo foi novamente submetido à apreciação do mesmo grupo de especialistas para avaliação quanto à pertinência e clareza, bem como a análise do layout. Nesta etapa, os 13 especialistas responderam os questionários.

Este estudo foi desenvolvido após apreciação e aprovação do Comitê de Ética em Pesquisa da Universidade Federal de Viçosa (Parecer Ético № 3.077.121). Aos especialistas incluídos na segunda, terceira e quarta etapas do estudo, foi enviada uma carta convite via e-mail, descrevendo o objetivo da pesquisa, e convidando-os a participar. Aos que aceitaram, foi solicitada a assinatura do TCLE.

\section{RESULTADOS}

A primeira etapa do estudo consistiu na identificação dos indicadores empíricos de avaliação das Necessidades Humanas Básicas em pacientes oncológicos através de busca na literatura. A Figura 2 mostra o fluxograma síntese da análise dos estudos que culminaram na extração final dos indicadores empíricos.

Figura 2 - Fluxograma do processo de seleção dos artigos que compuseram a amostra deste estudo. Viçosa, Minas Gerais - BR, 2020.

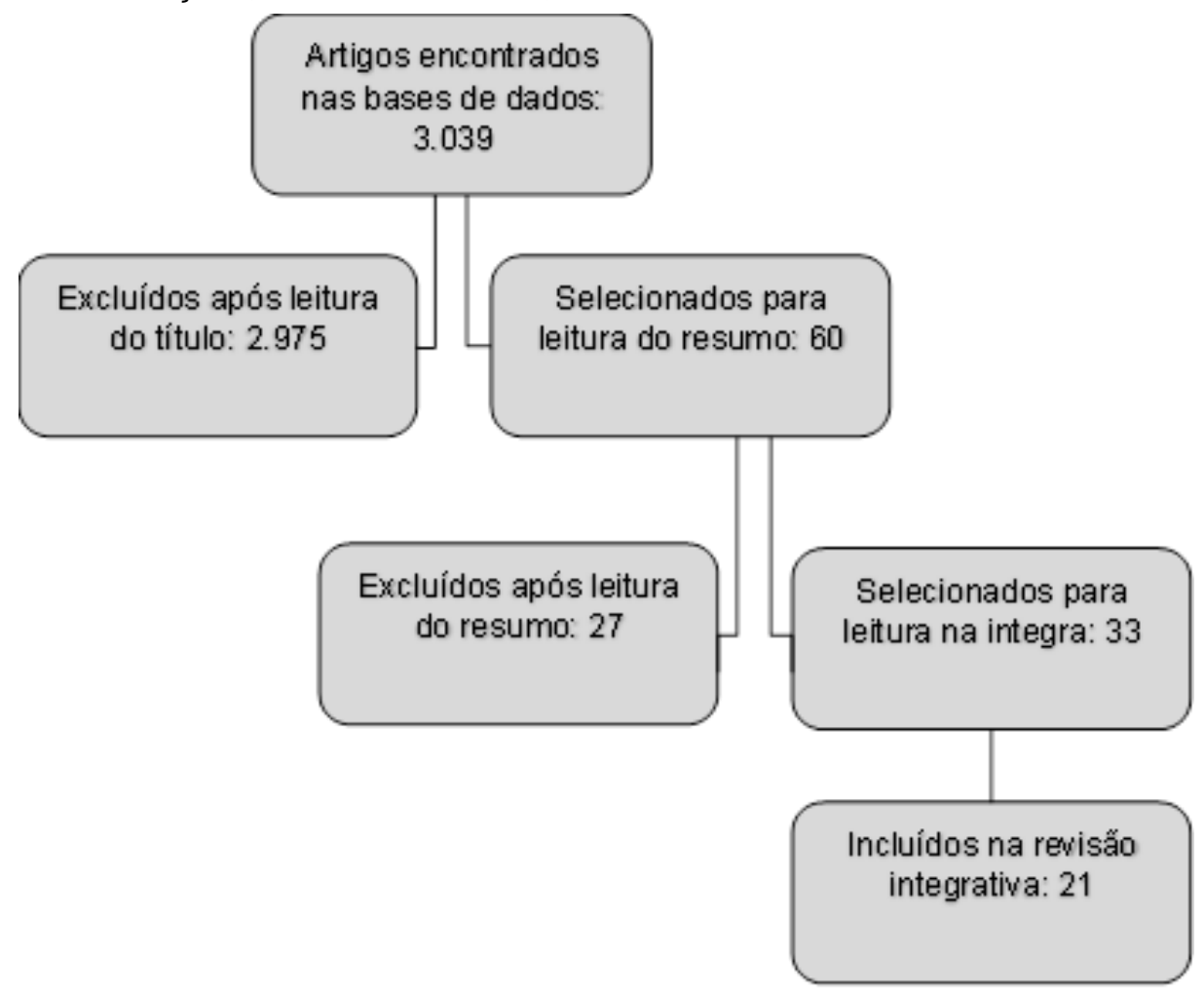

Fonte: Cheloni IG, et al., 2020. 
Após análise dos 21 estudos que compuseram a amostra, foram identificados 107 indicadores empíricos que representam os sinais e sintomas que devem ser utilizados para avaliar as necessidades humanas básicas afetadas em pacientes submetidos ao tratamento oncológico. Destes, 64 (59,81\%) referiam-se a indicadores de avaliação das necessidades psicobiológicas, $40(37,38 \%)$ a indicadores de avaliação das necessidades psicossociais, e $3(2,81 \%)$ a indicadores de avaliação das necessidades psicoespirituais. Não foram encontrados, na literatura consultada, indicadores empíricos para avaliar os constructos recreação e lazer, liberdade e participação, espaço, e criatividade das Necessidades Psicossociais. Quanto às Necessidades Psicobiológicas, não foram encontrados indicadores para avaliação do cuidado corporal e ambiental e segurança física e do meio ambiente.

Na segunda etapa do estudo foi realizada a validação dos indicadores empíricos junto ao comitê de especialistas. Dos 14 especialistas que participaram do estudo, $10(71,5 \%)$ eram do sexo feminino, com idade entre 27 e 52 anos. O ano da conclusão da graduação em enfermagem variou entre 1988 e 2016. O tempo de experiência profissional como enfermeiro variou de 02 a 30 anos (média de 11 anos), e da prática clínica em enfermagem oncológica variou entre 02 e 24 anos (média de 10 anos). Quanto à titulação, 08 (57,1\%) eram especialistas em Oncologia, 05 (35,6\%) Mestres; e 01(7,3\%) possuía Graduação em Enfermagem. Quanto à experiência com pesquisa, 01 (7,2\%) participava de pesquisas na pratica clínica, e 01 (7,2\%) já havia tido diagnóstico positivo para câncer e sido submetido à terapia antineoplásica.

Dos 107 indicadores empíricos identificados na literatura, os especialistas concordaram em $80 \%$ ou mais que 83 eram indicadores relevantes de serem avaliados em pacientes oncológicos. Destes, $52(60 \%)$ referiam-se a indicadores de avaliação das necessidades psicobiológicas, 30 (35,7\%) das necessidades psicossociais e $01(4,3 \%)$ da necessidade psicoespiritual. Os indicadores validados estão descritos no Quadro 2.

Ainda nesta etapa do estudo, foi sugerido pelos especialistas a inclusão de 58 novos indicadores empíricos que os mesmos consideraram como importantes de serem avaliados em pacientes submetidos à quimioterapia. Destes, $36(61,9 \%)$ eram relacionados à avaliação das necessidades psicobiológicas, dos quais destacam-se: prática de exercícios físicos, higiene corporal, déficit prévio para o autocuidado, percepção gustativa, e queixas na atividade sexual. Também foi sugerida a inclusão de 19 (32,7\%) indicadores relacionados à avaliação das necessidades psicossociais.

$\mathrm{Na}$ terceira etapa do estudo, foi feita a construção da versão preliminar do instrumento de coleta de dados, tendo por referência os indicadores validados e sugeridos na etapa dois do estudo. Esta versão preliminar do instrumento foi composta por 141 indicadores empíricos, sendo $88(62,4 \%)$ para avaliação das necessidades psicobiológicas, 49 (34,7\%) para avaliação das necessidades psicossociais, e 04 (02,9\%) para avaliação das necessidades psicoespirituais. Após análise dos especialistas, foram sugeridas 37 modificações sendo 19 inclusões, 10 exclusões e 08 modificações de nomes de itens, ou de localização dos mesmos dentro do instrumento. Os indicadores que compuseram a versão preliminar do instrumento de coleta de dados estão descritos no Quadro 3.

$\mathrm{Na}$ etapa 4, o instrumento de coleta de dados refinado após a etapa 3 do estudo foi novamente submetido à avaliação dos especialistas para a validação de conteúdo e de aparência da versão final do instrumento. Ao todo, nesta última rodada foram sugeridas 24 modificações na versão final do instrumento, sendo 08 inclusões, 5 exclusões e 11 modificações de nomes de itens, ou de localização dos mesmos dentro do instrumento.

A versão final do instrumento de coleta de dados para consulta de enfermagem a pacientes atendidos em ambulatório de quimioterapia ficou constituída por 167 itens, agrupados conforme a semelhança temática nos seguintes eixos:1-Identificação do cliente; 2- Diagnóstico; 3-Planejamento Terapêutico; 4-Condição atual de saúde; 5-Necessidades psicossociais; 6-Necessidades psicoespitituais; 7-Necessidades psicobiológicas; 8Solicitações do paciente/familiares; 9-Impressão do enfermeiro sobre o paciente/familiares/outros dados relevantes e 10- Encaminhamento ao profissional multidisciplinar. A Figura 3 mostra a versão final do instrumento de coleta de dados. 
Quadro 2 - Indicadores empíricos que obtiveram índice de concordância maior ou igual a 80\% pelos especialistas e foram considerados relevantes de serem avaliados em pacientes submetidos a quimioterapia. Viçosa, Minas Gerais, Brasil, 2020.

\begin{tabular}{|c|c|c|}
\hline \multicolumn{3}{|c|}{ Indicadores Psicossocial } \\
\hline Construto & Indicador Empírico & IC \\
\hline Comunicação & Capacidade de comunicação efetiva com os profissionais de saúde & 92,86 \\
\hline Gregária & $\begin{array}{l}\text { Desamparo familiar } \\
\text { Dificuldade de interação social } \\
\text { Sentimento de solidão }\end{array}$ & $\begin{array}{l}85,71 \\
85,71 \\
85,71\end{array}$ \\
\hline Segurança Emocional & $\begin{array}{l}\text { Insegurança em relação à qualidade de vida futura } \\
\text { Sofrimento } \\
\text { Insegurança quanto à possibilidade recidiva ou diagnóstico de um novo câncer } \\
\text { Estado de choque } \\
\text { Expectativa de melhora } \\
\text { Pensamento suicida } \\
\text { Sensação de impotência } \\
\text { Sentimento de culpa } \\
\text { Sentimento de esperança } \\
\text { Sentimento de fracasso } \\
\text { Sentimento de indignação } \\
\text { Sentimento de medo } \\
\text { Sentimento de raiva } \\
\text { Sentimento de tristeza } \\
\text { Sentimento de vergonha } \\
\text { Temor às mutilações e desfigurações }\end{array}$ & $\begin{array}{l}100 \\
92,86 \\
92,86 \\
85,71 \\
85,71 \\
85,71 \\
85,71 \\
85,71 \\
85,71 \\
85,71 \\
85,71 \\
85,71 \\
85,71 \\
85,71 \\
85,71 \\
85,71\end{array}$ \\
\hline Amor e Aceitação & $\begin{array}{l}\text { Autoimagem ou auto aceitação prejudicada } \\
\text { Negação da doença } \\
\text { Rejeição do (a) parceiro (a) }\end{array}$ & $\begin{array}{l}92,86 \\
85,71 \\
85,71\end{array}$ \\
\hline $\begin{array}{l}\text { Autoestima, Autoconfiança } \\
\text { e Autorrespeito }\end{array}$ & $\begin{array}{l}\text { Nível de Autonomia } \\
\text { Nível da autoestima }\end{array}$ & $\begin{array}{l}85,71 \\
85,71\end{array}$ \\
\hline $\begin{array}{l}\text { Educação para a Saúde/ } \\
\text { Aprendizagem }\end{array}$ & $\begin{array}{l}\text { Nível de conhecimento sobre a doença } \\
\text { Nível de conhecimento sobre o tratamento }\end{array}$ & $\begin{array}{c}100 \\
92,86\end{array}$ \\
\hline Auto Realização & $\begin{array}{l}\text { Impacto da doença nas atividades de vida diária } \\
\text { Impacto da doença na qualidade de vida } \\
\text { Capacidade de desempenhar suas atividades laborais }\end{array}$ & $\begin{array}{r}100 \\
100 \\
92,86\end{array}$ \\
\hline
\end{tabular}




\begin{tabular}{|c|c|c|}
\hline \multicolumn{3}{|c|}{ Indicador Psicoespiritual } \\
\hline \multicolumn{2}{|c|}{ Presença de crença ou religião } & 100 \\
\hline \multicolumn{3}{|c|}{ Indicadores Psicobiológico } \\
\hline \multirow{3}{*}{ Hábitos de vida } & Etilismo & 100 \\
\hline & Tabagismo & 100 \\
\hline & Uso de drogas & 100 \\
\hline \multirow{3}{*}{ Regulação Neurológica } & Estado Emocional & 100 \\
\hline & Nível de consciência & 100 \\
\hline & Reflexos motores e sensoriais (Tremores, tonturas, parestesias, paresias) & 100 \\
\hline \multirow{4}{*}{$\begin{array}{l}\text { Percepção de órgãos e } \\
\text { sentidos }\end{array}$} & Dor & 100 \\
\hline & Percepção auditiva & 100 \\
\hline & Percepção tátil & 92,86 \\
\hline & Percepção visual (fotossensibilidade) & 85,71 \\
\hline \multirow{3}{*}{ Oxigenação } & Presença de Dispneia & 100 \\
\hline & Presença de tosse & 100 \\
\hline & Ausculta respiratória & 92,86 \\
\hline \multirow{7}{*}{ Regulação Vascular } & Alterações na Pressão Arterial & 100 \\
\hline & Mielossupressão & 92,86 \\
\hline & Presença de Edema & 92,86 \\
\hline & Presença de Linfedema & 92,86 \\
\hline & Anemia & 85,71 \\
\hline & Hemoptise & 85,71 \\
\hline & Trombocitopenia & 85,71 \\
\hline Regulação Térmica & Presença de febre & 100 \\
\hline Hidratação & Alterações no estado de hidratação & 100 \\
\hline \multirow{5}{*}{ Alimentação } & Presença de distúrbios alimentares (Anorexia, desnutrição, obesidade, perda de peso) & $\frac{92,86}{100}$ \\
\hline & Queixas alimentares (disfagia, epigastralgia, náuseas, inapetência, xerostomia) & 100 \\
\hline & Mudança de hábitos alimentares & 100 \\
\hline & Dificuldade de deglutição & 92,86 \\
\hline & Ingesta nutricional inadequada & 85,71 \\
\hline
\end{tabular}




\begin{tabular}{|c|c|c|}
\hline Eliminação & $\begin{array}{l}\text { Distúrbios na eliminação urinária (oligúria, poliúria, disúria, anúria) } \\
\text { Distúrbios na eliminação intestinal (diarreia, flatos, constipação, incontinência fecal) } \\
\text { Característica da Urina } \\
\text { Característica das Fezes } \\
\text { Uso de medicamento com potencial de Nefrotoxicidade }\end{array}$ & $\begin{array}{r}100 \\
100 \\
100 \\
100 \\
92,86\end{array}$ \\
\hline Integridade Física & $\begin{array}{l}\text { Presença de estomas } \\
\text { Lesão em rede venosa } \\
\text { Áreas de hiperpigmentação da pele } \\
\text { Integridade das membranas mucosas } \\
\text { Lesão em mucosas }\end{array}$ & $\begin{array}{r}100 \\
100 \\
100 \\
100 \\
92,86\end{array}$ \\
\hline Sono e Repouso & $\begin{array}{l}\text { Alterações no sono (Insônia, sonolência durante o dia, incapacidade de repor as energias } \\
\text { após o sono) }\end{array}$ & 85,71 \\
\hline Atividade Física & $\begin{array}{l}\text { Fadiga } \\
\text { Dificuldade para deambular } \\
\text { Limitações nas atividades de vida diária } \\
\text { Prejuízo à funcionalidade dos membros } \\
\text { Redução da capacidade funcional }\end{array}$ & $\begin{array}{c}100 \\
100 \\
100 \\
92,86 \\
85,71\end{array}$ \\
\hline Sexualidade & $\begin{array}{l}\text { Queixas na atividade sexual (Disfunção erétil, libido comprometida) } \\
\text { Disfunção reprodutiva } \\
\text { Prejuízo na vida sexual } \\
\text { Sintomas do climatério }\end{array}$ & $\begin{array}{r}100 \\
92,86 \\
85,71 \\
85,71\end{array}$ \\
\hline $\begin{array}{l}\text { Regulação: Crescimento } \\
\text { vascular }\end{array}$ & Alopecia & 92,86 \\
\hline Terapêutica & $\begin{array}{l}\text { Necessidade de cuidados de apoio } \\
\text { Reações alérgicas } \\
\text { Dependente do profissional de saúde para resolução de seus problemas. }\end{array}$ & $\begin{array}{l}92,86 \\
92,86 \\
85,71\end{array}$ \\
\hline
\end{tabular}

Fonte: Cheloni IG, et al., 2020. 
Quadro 3 - Itens que compuseram a versão preliminar do instrumento de coleta de dados para consulta de enfermagem ao paciente oncológico ambulatorial, Viçosa, Minas Gerais, Brasil, 2020.

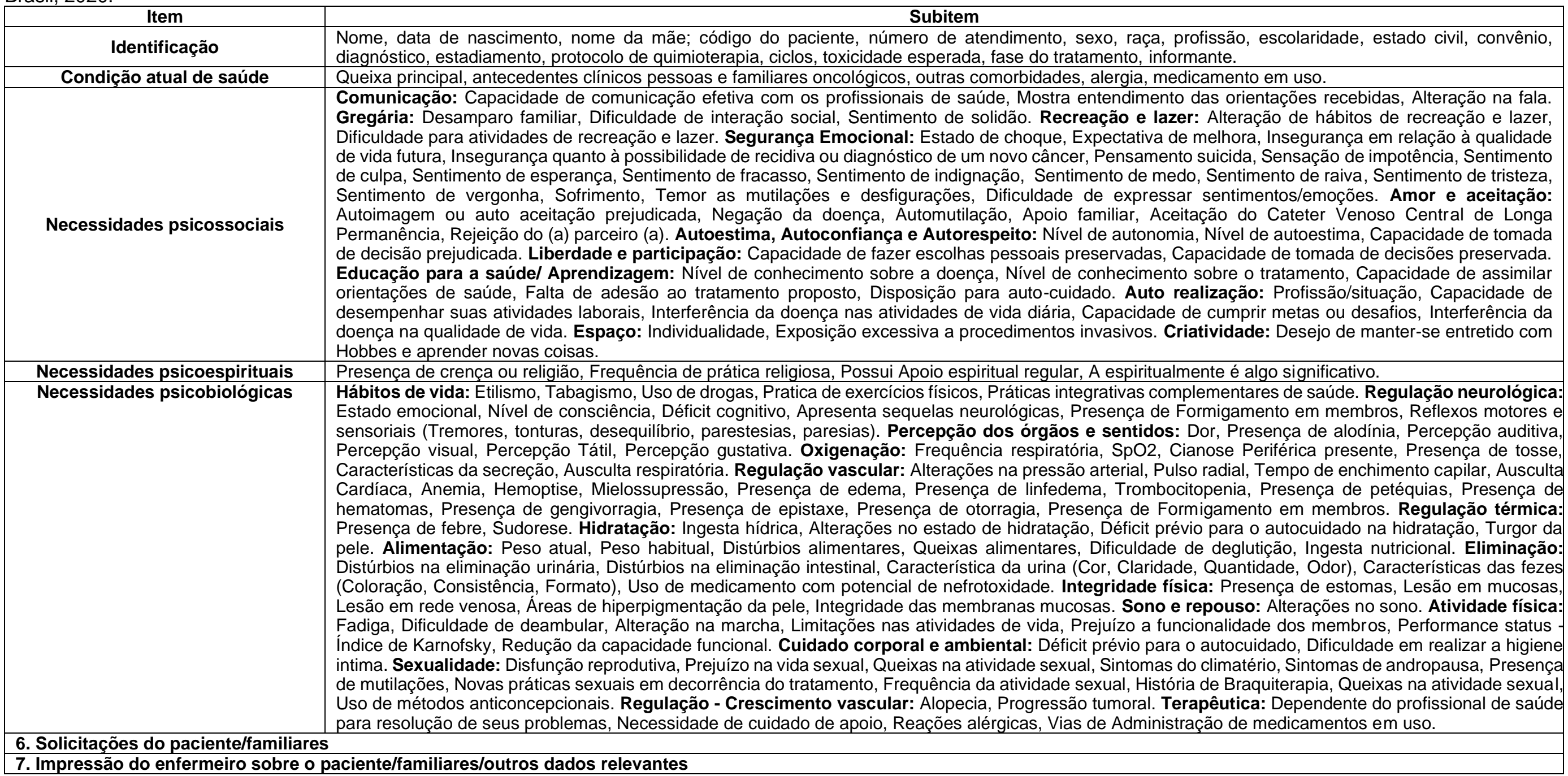

Fonte: Cheloni IG, et al., 2020. 
Figura 3 - Instrumento de coleta de dados de Enfermagem para pacientes submetidos a quimioterapia ambulatorial. Viçosa - MG, 2020.

\begin{tabular}{|c|c|}
\hline \multicolumn{2}{|c|}{$\begin{array}{l}\text { Instrumento de coleta de dados: } \\
\text { Ambulatório de Quimioterapia } \\
\text { Fundamentado na Teoria de Wanda Horta }\end{array}$} \\
\hline \multicolumn{2}{|r|}{1 - Identificação } \\
\hline Gênero: ( )M ( )F ( )Outro: & )F ( )Outro: Raça: \\
\hline \multicolumn{2}{|c|}{ Estado Civil: $\quad$ Cidade/Estado: $\quad$ Convênio: } \\
\hline \multicolumn{2}{|c|}{ E-mail: $\quad$ Telefone(s): } \\
\hline \multicolumn{2}{|c|}{ Informante: ( )Paciente ( )Membro da familia ( )Amigo ( )Profissional da Saúde ( )Outro: } \\
\hline \multicolumn{2}{|c|}{ 2- Diagnóstico } \\
\hline \multirow{2}{*}{\multicolumn{2}{|c|}{ 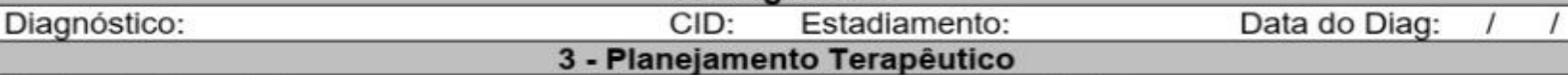 }} \\
\hline & \\
\hline \multicolumn{2}{|c|}{ Protocolo Terapêutico: $\quad$ Ciclos: } \\
\hline \multicolumn{2}{|c|}{ Fase do Tratamento: ( )Adjuvante ( )Neoadjuvante ( )Curativa ( )Paliativa } \\
\hline \multicolumn{2}{|c|}{ 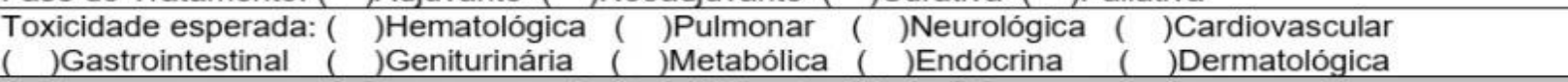 } \\
\hline \multirow{2}{*}{\multicolumn{2}{|c|}{$\begin{array}{l}\text { Queixa principal: } \\
\text { Antecedentes clinicos pessoais e familiares oncológicos: }\end{array}$}} \\
\hline & \\
\hline \multicolumn{2}{|c|}{$\begin{array}{l}\text { Outras comorbidades: } \\
\text { Alergia: ( )Não ( ) Sim: } \\
\text { Medicamentos em uso: }(\text { Não ( ) Sim Quais }\end{array}$} \\
\hline \multirow{2}{*}{\multicolumn{2}{|c|}{ 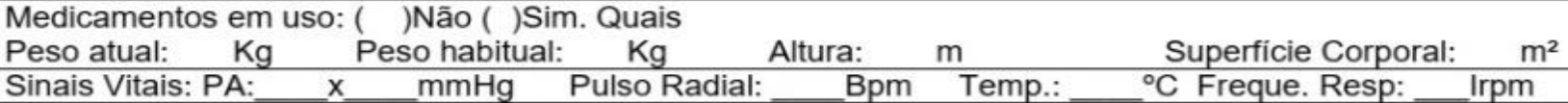 }} \\
\hline & \\
\hline \multicolumn{2}{|c|}{ 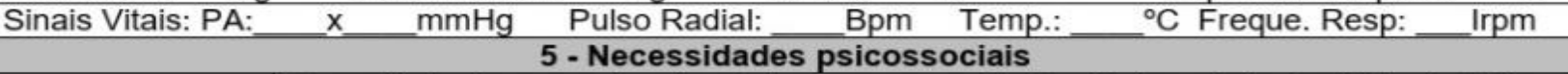 } \\
\hline Comunicação & $\begin{array}{l}\text { Capacidade de comunicação efetiva com os profissionais de saúde: ( )Não ( )Sim. } \\
\text { Mostra entendimento das orientações recebidas: ( )Não ( )Sim. } \\
\text { Alteração na fala: }(\text { ) Não ( ) Sim. Qual: }\end{array}$ \\
\hline Gregária & $\begin{array}{l}\text { Desamparo familiar: ( )Não ( )Sim. Dificuldade de interação social: ( )Não ( )Sim. } \\
\text { Sentimento de solidão: ( )Não ( ) Sim. }\end{array}$ \\
\hline $\begin{array}{l}\text { Recreação e } \\
\text { lazer }\end{array}$ & $\begin{array}{l}\text { Alteração de hábitos de recreação e lazer: ( )Não ( )Sim: } \\
\text { Mudanças nas atividades de Recreação e Lazer: ( )Não ( )Sim. }\end{array}$ \\
\hline $\begin{array}{l}\text { Segurança } \\
\text { Emocional }\end{array}$ & 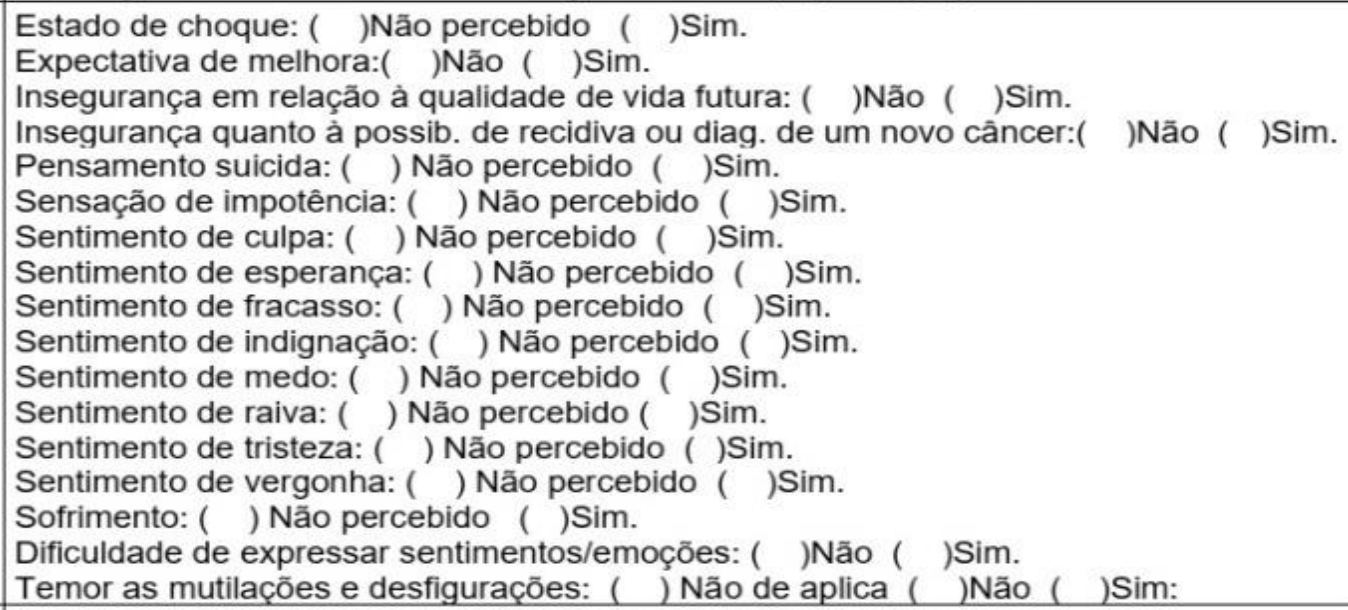 \\
\hline $\begin{array}{l}\text { Amor e } \\
\text { aceitação }\end{array}$ & $\begin{array}{l}\text { Autoimagem ou auto aceitação prejudicada: ( )Não ( )Sim. } \\
\text { Negação da doença: ( )Não ( )Sim. } \\
\text { Automutilação: ( )Não ( )Sim. Apoio familiar: ( )Não ( )Sim. } \\
\text { Aceitação de dispositivos decorrentes do tratamento: ( )Não ( )Sim. } \\
\text { Rejeição do (a) parceiro (a): ( )Não( )Sim. }\end{array}$ \\
\hline $\begin{array}{l}\text { Autoestima, } \\
\text { Autoconfiança e } \\
\text { Autorespeito }\end{array}$ & $\begin{array}{l}\text { Nivel de autonomia: ( )Não comprometida ( )Comprometida: } \\
\text { Nivel de autoestima: ( )Não comprometida ( )Comprometida: } \\
\text { Capacidade de tomada de decisão prejudicada: ( )Não ( ) Sim. }\end{array}$ \\
\hline $\begin{array}{l}\text { Educação para } \\
\quad \text { a saúdel } \\
\text { Aprendizagem }\end{array}$ & $\begin{array}{l}\text { Nivel de conhecimento sobre a doença: ( )Adequado ( ) Inadequado ( )Nenhum. } \\
\text { Nivel de conhec. sobre o tratamento: ( )Adequado ( ) Inadequado ( )Nenhum. } \\
\text { Capac. de assimilar orientações de saúde: ( )Adequada ( )Inadequada ( )Nenhuma. } \\
\text { Adesão ao tratamento proposto: ( )Não ( )Sim. } \\
\text { Disposição para autocuidado: ( )Não ( ) Sim. }\end{array}$ \\
\hline
\end{tabular}




\begin{tabular}{|c|c|}
\hline Auto realização & $\begin{array}{l}\text { Mantém atividade profissional: ( )Não ( ) Sim. Qual: } \\
\text { Capacidade de desempenhar suas atividades laborais: ( )Não ( ) Sim. } \\
\text { Interfência da doença nas atividades de vida diária: ( )Não ( )Sim. Cite: } \\
\text { Capacidade de cumprir metas ou desafios: ( )Não ( ) Sim. } \\
\text { Interferência da doença na qualidade de vida: ( )Não ( )Sim: }\end{array}$ \\
\hline Espaço & $\begin{array}{l}\text { Individualidade:( )Não comprometida ( )Comprometida: } \\
\text { Exposição excessiva a procedimentos invasivos: ( Não ( ) Sim. } \\
\text { Está adaptado aos dispositivos decorrentes do tratamento: ( )Não ( ) Sim. }\end{array}$ \\
\hline Criatividade & Desejo de manter-se entretido e aprender novas coisas: ( )Não ( )Sim: \\
\hline & $6-$ Necessidades psicoespirituais \\
\hline $\begin{array}{l}\text { Espiritualidade } I \\
\text { Crença }\end{array}$ & $\begin{array}{l}\text { Presença de crença ou religião: ( ) Não ( )Sim. Qual? } \\
\text { Freq. de prática religiosa:( )Esporádico ( )1x/sem ( )2 a 3x/sem ( ) +3x/sem. } \\
\text { Possui Apoio espiritual regular: ( )Não ( ) Sim. Qual? } \\
\text { A espiritualmente é algo significativo: ( )Não ( ) Sim. }\end{array}$ \\
\hline & 7 - Necessidades Psicobiológicas \\
\hline Hábitos de vida & $\begin{array}{l}\text { Etilismo: ( )Não ( )Sim. Tabagismo: ( )Não ( )Sim. } \\
\text { Uso de drogas ilícitas: ( )Não ( )Sim: Prática de exer físicos: ( )Não ( ) Sim: } \\
\text { Práticas integrativas complementares de saúde: }(\text { Não ( ) Sim: }\end{array}$ \\
\hline $\begin{array}{l}\text { Regulação } \\
\text { neurológica }\end{array}$ & $\begin{array}{l}\text { Estado emoc: ( )Agressivo ( )Agitado ( )Ansioso ( )Alegre ( ) Calmo ( )Depressivo. } \\
\text { Nivel de consciência: ( )Consciente ( )Confuso. } \\
\text { Apresenta sequelas neurológicas: ( )Não ( )Sim: } \\
\text { Alteração de reflexos motores e sensoriais:( )Não ( )Sim: ( )Tremores ( } \\
\text { )Tonturas, ( )Desequilibrio ( )Parestesias ( )Paresias. }\end{array}$ \\
\hline $\begin{array}{l}\text { Percepção dos } \\
\text { órgâos e } \\
\text { sentidos }\end{array}$ & $\begin{array}{l}\text { Dor: ( )Não ( )Sim. Local: } \\
\text { Percepção auditiva: ( )Não comprometida ( )Comprometida } \\
\text { Percepção visual (Fotossensibilidade): ( )Não comprometida ( ) Comprometida } \\
\text { Percepção Tátil: ( )Não comprometida ( )Comprometida } \\
\text { Percepção gustativa: ( )Não comprometida ( )Comprometida }\end{array}$ \\
\hline Oxigenação & $\begin{array}{l}\text { SpO2:- ( ) Eupneico ( )Taquipneico ( )Bradipneico ( )Dispneico. } \\
\text { Cianose periférica presente: ( )Não ( )Sim: } \\
\text { Presença de tosse: ( )Não ( )Sim. Características: ( )Seca ( )Produtiva. } \\
\text { Características da secreção: Coloração: } \\
\text { Volume: }\end{array}$ \\
\hline $\begin{array}{l}\text { Regulação } \\
\text { vascular }\end{array}$ & 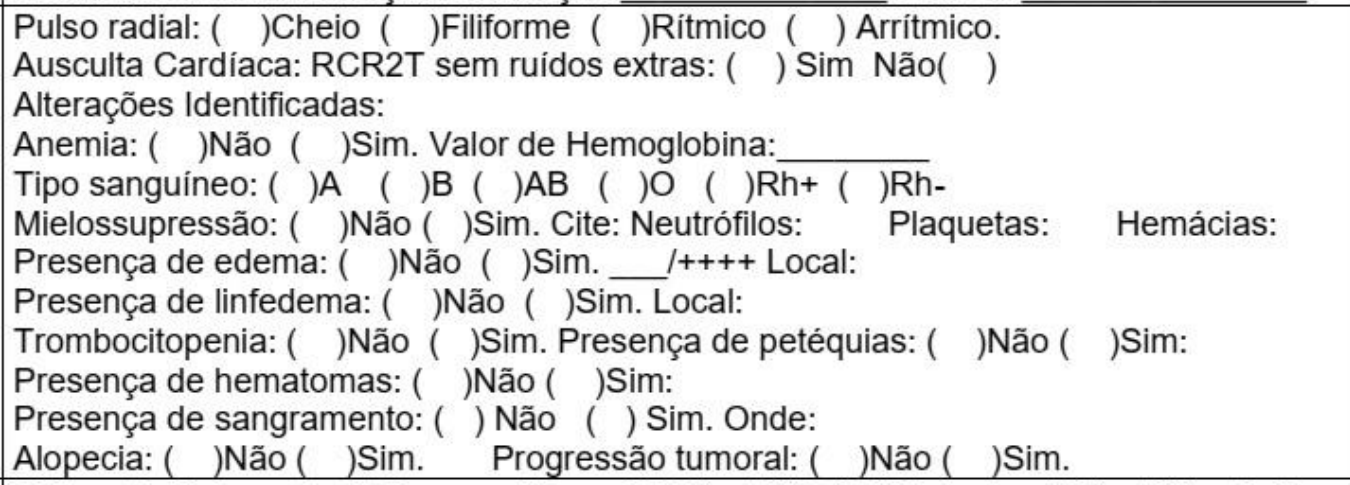 \\
\hline $\begin{array}{l}\text { Regulação } \\
\text { térmica }\end{array}$ & $\begin{array}{l}\text { Febre aferida ou percebida nas ultimas 48h? ( )Não ( )Sim.___ }{ }^{\circ} \mathrm{C}(\text { )Não aferida. } \\
\text { Sudorese ( )Não ( )Sim Tipo: }\end{array}$ \\
\hline Hidratação & $\begin{array}{l}\text { Ingesta hídrica: ( )Não comprometida ( )Comprometida: } \\
\text { Alterações no estado de hidratação: ( )Não ( )Sim: } \\
\text { Déficit prévio para o autocuidado na hidratação: ( )Não ( )Sim: } \\
\text { Turgor da pele: ( )Preservado ( )Diminuído ( ) Ressecado }\end{array}$ \\
\hline Nutrição & 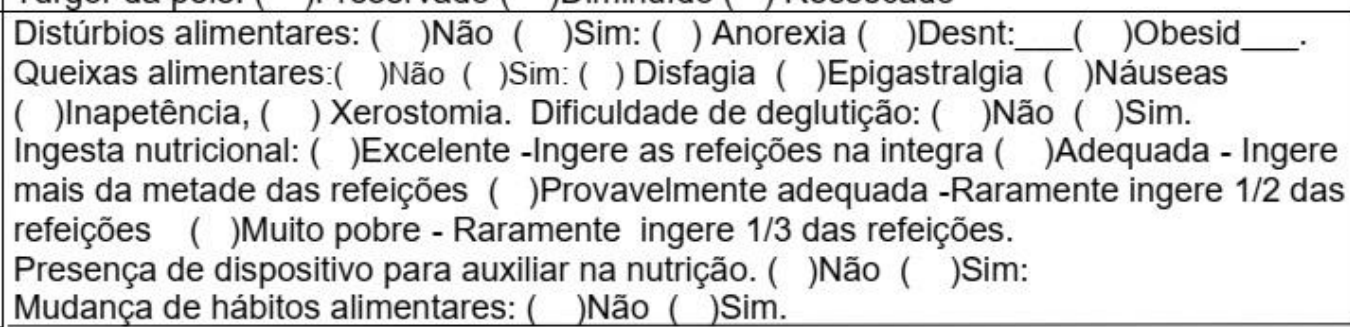 \\
\hline
\end{tabular}




\begin{tabular}{|c|c|}
\hline Eliminação & $\begin{array}{l}\text { Distúrbios na eliminação urinária:( )Não ( )Sim: ( ) Oligúria ( )Poliúria ( ) Disúria } \\
\text { ( )Anúria ( )Incontinência Frequência urinária : x dia } \\
\text { Distúrbios na eliminação intestinal:( )Não ( )Sim: ( )Diarréia ( )Constipação } \\
\text { ( )Flatos ( )Incontinência ( )Dor: Frequência de eliminação: x dia } \\
\text { Característica da urina: Cor: ( ) Limpida ( ) Concentrada ( )Hematúria } \\
\text { Claridade: ( ) Turva ( ) Transparente. Quantidade: ( )500 a 1700ml/dia ( )400mlou- } \\
\text { ( )1800ml ou+. Odor:( )Sui generis ( )Amoniacal ( )Adocicado ( )Pútrido. } \\
\text { Características das fezes: Coloração:( )Esverdeada ( )Acinzentada ( )Enegrecidas } \\
\text { ( )Amareladas ( )Amarronzadas. Presença de sangue vivo nas fezes: ( )Não ( )Sim. } \\
\text { Consistência:( )Consistente ( )Pastosa ( )Liquida-pastosa ( )Liquida. } \\
\text { Formato: ( )1.Caroços duros e separados, como nozes (dificil de passar) ( )2.Forma de } \\
\text { salsicha, mas granuloso ( )3.Como uma salsicha, mas com fissuras em sua superficie } \\
\text { ( )4.Como uma salsicha ou serpente, suave e macio ( )5.Bolhas suaves com bordas } \\
\text { nítidas (que passa facilmente) ( )6.Peças fofas com bordas em pedaços ( )7.Aquoso, } \\
\text { sem partes sólidas, inteiramente líquido. } \\
\text { Uso de medicamento com potencial de nefrotoxidade: ( )Não ( )Sim. Cite: } \\
\text { Presença de dispositivo para auxiliar na eliminação. ( )Não ( )Sim: }\end{array}$ \\
\hline $\begin{array}{l}\text { Integridade } \\
\text { física }\end{array}$ & $\begin{array}{l}\text { Presença de estomas: ( )Não ( )Sim Local: } \\
\text { Lesão em mucosas: ( )Não ( )Sim Local: } \\
\text { Lesão em rede venosa: ( )Não ( )Sim Local: } \\
\text { Rede venosa adequada para tratamento proposto: ( )Sim ( )Não: } \\
\text { Áreas de hiperpigmentação da pele: ( )Não ( )Sim: } \\
\text { Integridade das membranas mucosas: ( )Não ()Sim: } \\
\text { Presença de Lesão por Pressão: ( ) Não ( ) Sim. Estagio: Local: } \\
\text { Presença de drenos ou cateteres: ( )Não ( )Sim. Qual: }\end{array}$ \\
\hline Sono e repouso & $\begin{array}{l}\text { Alterações no sono:( ) Não ( )Sim: ( )Insônia ( )Sonolência durante o dia } \\
\text { ( ) Incapacidade de repor as energias após o sono. }\end{array}$ \\
\hline Atividade física & $\begin{array}{l}\text { Fadiga: ( )Não ( )Sim. Dificuldade de deambular: ( )Não ( )Sim. } \\
\text { Alteração na marcha:( )Não ( )Sim. Limitações nas ativi de vida:( )Não ( )Sim. } \\
\text { Prejuizo a funcionalidade dos membros: ( )Não ( )Sim. İndice de Karnofsky: } \% \text {. } \\
\text { Redução da capacidade funcional: ( )Não ( )Sim. } \\
\text { Utiliza dispositivo para auxiliar na deambulação: ( )Não ( )Sim: }\end{array}$ \\
\hline $\begin{array}{l}\text { Cuidado } \\
\text { corporal e } \\
\text { ambiental }\end{array}$ & $\begin{array}{l}\text { Déficit para o autocuidado: Higiene corporal ( )Não ( )Sim. } \\
\text { Déficit para o autocuidado: Higiene oral ( )Não ( )Sim. } \\
\text { Dificuldade em realizar a higiene intima: ( )Não ( )Sim. } \\
\text { Tipo de precaução: ( ) Padrão ( ) Contato ( ) Aerossóis ( ) Gotículas ( ) } \\
\text { Contato e aerossóis ( ) Contato e Gotícula. }\end{array}$ \\
\hline Sexualidade & 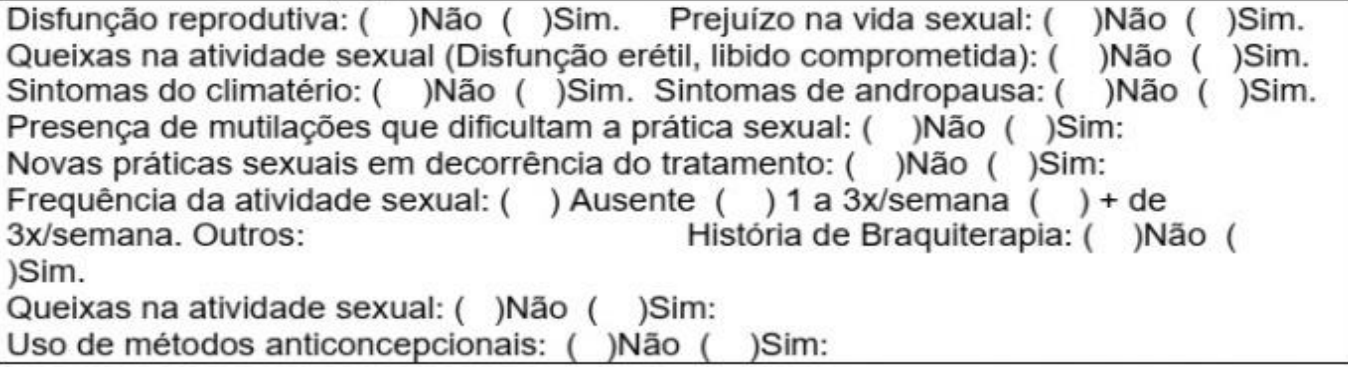 \\
\hline Terapêutica & $\begin{array}{l}\text { Dependente do profis. de saúde para resolução de seus problemas: ( )Não ( )Sim. } \\
\text { Necessidade de cuidado de apoio: ( )Não ( )Sim. } \\
\text { Histórico de reação transfusional: ( )Não ( ) Sim. }\end{array}$ \\
\hline \multicolumn{2}{|r|}{8 - Solicitações do paciente/familiares } \\
\hline \multicolumn{2}{|r|}{9 - Impressão do enfermeiro sobre o paciente/familiares/outros dados relevantes: } \\
\hline \multicolumn{2}{|c|}{ Riscos identificados: ( ) Não （ ） Sim. Qual/Quais: } \\
\hline \multicolumn{2}{|c|}{$10-$ Encaminhamento ao profissional multidisciplinar } \\
\hline \multicolumn{2}{|c|}{$\begin{array}{l}\text { ( ) Não ( ) Sim. Qual: } \\
\text { Justifique: }\end{array}$} \\
\hline
\end{tabular}

Fonte: Cheloni IG, et al., 2020. 


\section{DISCUSSÃO}

Este estudo objetivou a construção e validação de um instrumento de coleta de dados para consulta de Enfermagem para pacientes submetidos a quimioterapia ambulatorial, tendo por referencial teórico a teoria das Necessidades Humanas Básicas de Wanda Horta. Na primeira etapa do estudo, apenas 21 trabalhos eram relacionados ao cuidado de Enfermagem de pacientes em quimioterapia, especialmente a ambulatorial, o que aponta para uma lacuna na literatura acerca desta temática. Esta dificuldade foi relatada por outros autores ao tentarem fazer o mesmo levantamento para a construção de instrumento para coletar dados em pacientes idosos com câncer em tratamento paliativo para consulta de enfermagem em quimioterapia ambulatorial (TOLENTINO GS, et al., 2019; LANCKER AV, et al., 2015). Estes achados apontam para a necessidade de se pesquisar acerca da assistência de Enfermagem ao paciente oncológico em tratamento quimioterápico ambulatorial.

As preocupações evidenciadas por apontamentos dos especialistas quanto à validação da aparência e conteúdo da ferramenta de coleta de dados, corroboram com achados da literatura que afirmam a importância dos registros em todas as instâncias do processo de enfermagem (TOLENTINO GS, et al., 2019).

Os registros de enfermagem fornecem dados que ajudam no estabelecimento de planos de cuidados e na elaboração da evolução de enfermagem, atuando como subsídio para a Sistematização da Assistência de Enfermagem, além de ser um fator imprescindível para continuidade da assistência de qualidade, com menor índice de eventos adversos e maior segurança para o paciente (FERREIRA LL, et al., 2020).

Cabe lembrar que a comunicação efetiva é uma das metas internacionais de segurança do paciente. Deste modo, a utilização de um instrumento sistematizado de coleta de dados para guiar a consulta de Enfermagem e para documentar os achados, é uma ferramenta poderosa de comunicação da equipe multidisciplinar acerca dos achados que indicam as alterações nas necessidades humanas básicas dos pacientes oncológicos em tratamento quimioterápico ambulatorial, bem como para indicar as necessidades de cuidado. A elaboração de ferramentas que consigam extrair do paciente um número maior de informações, possibilitam um cuidado mais centralizado para o paciente, familiar e equipe multiprofissional (LEMOS DMP, et al., 2019).

A sugestão dos especialistas da inserção de subitens como peso, altura e superfície corporal do paciente somados aos subitens "protocolo de quimioterapia" e "número de ciclos" demonstra a preocupação dos mesmos com a dosagem da medicação prescrita pelo médico, uma vez que essa, está diretamente relacionada a superfície corporal do paciente. Estudo de revisão de escopo apontou algumas medidas para propiciar a segurança do paciente na administração da quimioterapia via parenteral. Dentre elas destacamse esquema quimioterápico; número do ciclo; todos os medicamentos listados empregando nomes genéricos completos; dose do fármaco escrita; dados para cálculo da dose com a descrição das variáveis usadas: peso, altura, superfície corpórea (OLIVEIRA PP, et al., 2019).

A manifestação dos especialistas quanto à duplicidade do subitem "Mostra entendimento das orientações recebidas", solicitando a remoção do mesmo de um constructo e o mantendo em outro, demonstra a preocupação em garantir que o paciente/familiar compreenda todas as informações oferecidas, visando um cuidado integral ao paciente oncológico. Os profissionais de saúde devem oferecer informações consistentes aos pacientes, especialmente sobre o gerenciamento dos efeitos colaterais adversos da quimioterapia no seu regresso a casa (SUWANKHONG D e LIAMPUTTONG P, 2019). Assim torna-se importante 0 empoderamento do paciente com câncer, incluindo-o no processo de cuidado. Ações como estas reduzem a ansiedade, possibilitam a aquisição de confiança e melhoram a adesão às/ao orientações/tratamento (TOLENTINO GS, et al., 2019).

Verificou-se a preocupação dos peritos em manter um instrumento enxuto e aplicável. Chamou a atenção dos pesquisadores a indicação dos especialistas de remoção dos itens "Possui Apoio espiritual regular" e "A espiritualidade é algo significativo" destinados à avaliação das necessidades psicoespirituais. Tais achados vão ao encontro de pesquisa realizada cujos achados mostraram o despreparo de alguns profissionais em lidar com as necessidades emocionais, sociais e espirituais. Pôde-se constatar que $32,5 \%$ dos profissionais frequentemente não oferecem suporte emocional para o paciente/familiar (PAPASTAVROU E, et al., 2016). 
Em contrapartida, autores relatam que a presença da espiritualidade no processo de adoecer por câncer vem sendo cada vez mais estudada, e evidências apontam que ela contribui positivamente com o paciente no enfrentamento da enfermidade e em sua qualidade de vida (BIRK MN, et al., 2019). Sendo assim a espiritualidade representa uma fonte de apoio que possibilita às pessoas sentirem-se mais amparadas, confiantes, esperançosas e motivadas.

Nesta pesquisa, foi ressaltada a importância de se incluir no instrumento o item destinado à avaliação da adequação da rede venosa para o tratamento quimioterápico proposto. A condição do cliente em uso de antineoplásico, os equipamentos utilizados na administração, bem como as propriedades dos agentes antineoplásicos infundidos caracterizam-se como fatores de risco para o extravasamento (DIAS SRS, et al., 2019). Sendo assim, o enfermeiro deve garantir que a via de administração do quimioterápico seja segura, o que requer avaliação criteriosa antes, durante a após a sua administração (RIBEIRO TS e SANTOS VO, 2015; REIS LAR et al., 2020).

Outro aspecto que chamou a atenção foi a sugestão de inclusão no instrumento de coleta de dados de um espaço para registro da necessidade do encaminhamento do paciente para atendimento com outro membro da equipe multidisciplinar. Esta sugestão vai ao encontro de estudos que definem o câncer como uma doença multifatorial, que exige uma abordagem multidisciplinar em todas as fases do tratamento, visando um tratamento eficaz, e contribuindo para a melhora da qualidade de vida dos pacientes. Uma assistência provida por uma equipe multidisciplinar visa a melhoria da qualidade de vida da pessoa e dos seus familiares frente a uma doença que ameace a vida, prevenindo e aliviando complicações oriundas do tratamento (SILVA MSJ, 2019; CAVEIÃO C, et al., 2019).

\section{CONCLUSÃO}

O estudo permitiu a construção e validação de um instrumento para coleta de dados para ser utilizado em pacientes submetidos a quimioterapia ambulatorial, baseado na Teoria das Necessidades Humanas Básicas de Wanda Horta. Este instrumento almeja contribuir para a coleta de dados de Enfermagem em ambulatórios de quimioterapia, conferindo maior visibilidade às ações de enfermagem, valorização da profissão e, principalmente, excelência no cuidado ao paciente oncológico. Por fim, este instrumento servirá como uma ferramenta norteadora para novas pesquisas nas áreas da assistência de enfermagem ao paciente oncológico, especialmente àqueles em quimioterapia ambulatorial.

\section{AGRADECIMENTOS E FINANCIAMENTO}

Este artigo é parte da dissertação de mestrado profissional de Igor Guerra Cheloni pela Universidade Federal de Viçosa - UFV, e foi financiado com bolsa de iniciação científica pelo Conselho Nacional de Desenvolvimento Científico e Tecnológico (CNPQ).

\section{REFERÊNCIAS}

1. BENEDET SA, BUB MBC. Manual de Diagnóstico de Enfermagem: uma abordagem baseada na Teoria das Necessidades Humanas e na Classificação Diagnóstica da NANDA. 2. ed. Florianópolis: Bernúncia, $2001 ; 220$.

2. BIRCK NM, et al. Perception of women with breast cancer about the care of Nursing to spirituality. Cienc. Cuid. Saúde [Internet]. 20 de fevereiro de 2019.

3. BONASSA EMA, GATO MIR. Terapêutica Oncológica para enfermeiros e farmacêuticos.4ª .ed. São Paulo: Atheneu, $2012 ; 627$.

4. BRAY F, et al. Global cancer statistics 2018: GLOBOCAN estimates of incidence and mortality worldwide for 36 cancers in 185 countries. CA: a cancer journal for clinicians, Hoboken, 2018; 68(6): 394-424.

5. CAVEIÃO C, et al. Ações do enfermeiro em cuidados paliativos na oncologia: uma revisão integrativa. Revista Saúde e Desenvolvimento [Internet], 2019; 13(16).

6. CHELONI GI, et al. Necessidades humanas básicas afetadas em pacientes oncológicos: revisão integrativa da literatura. Hu rev [Internet], 2020; 46: 1-11.

7. COFEN. Conselho Federal de Enfermagem. Resolução no. 358, de 15 de outubro de 2009. Sistematização da Assistência de Enfermagem e implementação do Processo de Enfermagem em ambientes públicos ou privados, Brasília, 2009.

8. DIAS SRS, et al. Padrões de cuidados em prevenção e tratamento de extravasamento de antineoplásicos baseado em evidências clínicas. Revista Enfermagem Atual InDerme, [Internet] 2019; 87(Edição Esp): 1-10. 
9. FEHRING RJ. Methods to validate nursing diagnoses. Heart and Lung, [S.I.], 1987; 16(6): 625-9.

10. FERREIRA LL, et al. Analysis of records by nursing technicians and nurses in medical records. Rev. Bras. Enferm. 2020; 73(2): 1-6.

11. GALVÃO CM. Níveis de evidências [editorial]. Acta Paul Enferm [Internet], 2006; 19(2).

12. INCA. Estimativa 2020: incidência de câncer no Brasil. Instituto Nacional de Câncer José Alencar Gomes da Silva. Coordenação de Prevenção e Vigilância. Rio de Janeiro: INCA, 2019; 122.

13. LANCKER AV, et al. An instrument to collect data on frequency and intensity of symptoms in older palliative cancer patients: a development and validation study. Eur J Oncol Nurs. 2016; (21): 38-47.

14. LEMOS DMP, et al. Comunicação efetiva para o cuidado seguro ao paciente com implante de dispositivo de assistência ventricular. Rev Gaúcha Enferm. [Internet]. 2019; 40(esp).

15. LYNN, MR. Determination and quantification of content validity. Nursing Research, [S.I.].1986; 34(6): $382-85$.

16. MENDES KDS, et al. Revisão integrativa: método de pesquisa para a incorporação de evidências na saúde e na enfermagem. TextoContextoEnferm. 2008; 17(4): 758-64.

17. OLIVEIRA PP, et al. Patient safety in the administration of antineoplastic chemotherapy and of immunotherapics for oncological treatment: scoping review. Texto contexto - enferm. [Internet]. 2019; 28: e20180312.

18. PAPASTAVROU E, et al. To what extent are patients' needs met on oncology units? The phenomenon of care rationing. Eur J Oncol Nurs [Internet]. 2016; 21: 48-56.

19. POLIT DF, BECK CT. Fundamentos da Pesquisa em Enfermagem. 7. ed. Porto Alegre: Artmed, $2011 ; 456$.

20. RAMALHO, JMN. Construção e validação de instrumento para coleta de dados de enfermagem em adultos de uma unidade de tratamento intensivo. Dissertação (Mestrado em Enfermagem). Centro de Ciências da Saúde da Universidade Federal da Paraíba, 2010; 133.

21. REIS LAR, et al. Consulta sistematizada de enfermagem em quimioterapia antineoplásica / Consulta sistematizada de enfermagem em quimioterapia Antineoplásica. Braz. J. of Develop., 2020; 6(2): 7668-83.

22. RIBEIRO TS, SANTOS VO. Segurança do Paciente na Administração de Quimioterapia Antineoplásica: uma Revisão Integrativa. Rev. Brasileira de Cancerologia [Internet]., 2015; 61(2): 145-53.

23. RODRIGUES SMN. Construção e validação de um instrumento de coleta de dados para pacientes oncohematológicos em tratamento quimioterápico ambulatorial. Dissertação (Mestrado em Enfermagem). Gestão em Saúde da Universidade Estadual do Ceará, Fortaleza 2017;118p.

24. SILVA MJS. Contribuições do farmacêutico para a equipe multiprofissional de terapia antineoplásica. Revista Brasileira de Farmácia Hospitalar e Serviços De Saúde, 2019; 5(3).

25. SOUZAAPMA, et al. Indicadores empíricos para a estruturação de um instrumento de coleta de dados em unidade cirúrgica. Rev. Eletr. Enf, 2009; 11(3): 501-8.

26. SUWANKHONG D, LIAMPUTTONG P. Physical and Emotional Experiences of Chemotherapy: a Qualitative Study among Women with Breast Cancer in Southern Thailand. Asian Pac J Cancer Prev, 2019; (2): 521-28.

27. TANNURE MC, PINHEIRO AM. SAE - Sistematização da Assistência de Enfermagem: Guia Prático. 3th. Rio de Janeiro: Guanabara Koogan; 2019; 340.

28. TOLENTINO GS, et.al. Construção e validação de instrumento para consulta de enfermagem em quimioterapia ambulatorial. Rev. Bras. Enferm, 2019; 72(2): 391-99.

29. VIDIGAL PD, et.al. Principais necessidades humanas básicas afetadas em pacientes com câncer e tromboembolismo venoso: revisão integrativa. Revista Enfermagem Atual In Derme, 2017; (Edição Especial): 113-119. 